http://dx.doi.org/10.18778/2196-8403.2019.02

SUSANNE KAUL

\title{
Was leistet die Kälte des Schauspielers bei LESSING und DIDEROT?
}

In diesem Beitrag geht es um eine schauspieltheoretische Debatte, die im 18. Jahrhundert aufgekommen ist und an der sich auch DIDEROT und LESSING beteiligt haben. Soll ein Schauspieler seine ganze Leidenschaft in eine Rolle legen und sein, was er darstellt, oder soll er distanziert und beherrscht die Rolle spielen, ohne darin aufzugehen? Während DIDEROT viele Gründe für den kalten Schauspieler ins Feld führt, plädiert LESSING für eine Mischung aus Feuer und Kälte. Die These dieses Beitrags ist, dass die Kälte bei LESSING im Dienste einer aufklärerischen Common Sense Moral steht, die in der Nähe zu Adam Smith gesehen werden kann, während DiDEROTS Verteidigung der Kälte ästhetisch begründet ist und allein der Kunstfertigkeit der Darstellung dienen soll. In einem Ausblick werden einige Implikationen dieser Statements über kalte und heiße Gefühle kritisch mit der Emotionstheorie der Gegenwart in Beziehung gesetzt.

\section{The Impact of "Cold Acting" in LESSING and DIDEROT}

This article presents the debate on theatre theory that arose in the 18th century and in which DiDEROT and LESSING participated. The conflict revolved around the question whether an actor should convey authentic emotions and identify with the character he/she plays or whether he/she should act in an unemotional and controlled way without becoming absorbed in the role. While DIDEROT delivers many arguments for "the cold actor", LESSING pleads for a mix of "fire" and "coldness". The thesis of this article is that LESSING's "coldness" is justified by an Enlightenment common sense morality that can be seen as akin to Adam Smith's ideas, while DIDEROT defends the "cold" way of acting in the name of aesthetic values which serve to assure a high artistic level of performance. The article looks at how some implications of these statements about "cold" and "hot" feelings are critically related to contemporary emotion theories. 


\section{Co sprawia aktorska ,gra na zimno’ u LESSINGA i DIDEROTA?}

$\mathrm{W}$ artykule przedstawiona jest debata dotycząca zasad gry aktorskiej, prowadzona w XVIII w. Brali w niej udział m.in. Diderot i LeSSING. Spór dotyczył pytania, czy aktor powinien przekazywać autentyczne emocje i identyfikować się z przedstawianą postacią czy też powinien grać w sposób zdystansowany i opanowany, bez stapiania się z rolą. DIDEROT podawał wiele argumentów na rzecz ,chłodnego' aktora, natomiast LESSING był zwolennikiem połączenia ognia i chłodu. Teza artykułu dotyczy funkcji ,gorącej' lub ,chłodnej’ gry: chłód służy w wypowiedziach LESSINGA oświeceniowemu Common Sense Moral. Jest to teoria bliska tezom Adama Smitha. DiDERoT natomiast broni chłodu w imię wartości estetycznych, służących poziomowi artystycznemu przedstawienia. Spojrzenie w pooświeceniową przyszłość wiąże wnioski z tez o chłodnych i gorących uczuciach ze współczesnymi teoriami o emocjach.

Schauspieler können Tränen für nichts fließen lassen. Am Ende des zweiten Akts bittet Hamlet einen Schauspieler, einen Monolog zu rezitieren, in dem von Hekubas Trauer um ihren Gatten Priamus erzählt wird. Hamlet ist bestürzt von der paradoxen Fähigkeit des Schauspielers, Tränen über das Schicksal einer Königin zu vergießen, die ihm real nichts bedeuten kann, weil sie nur im Mythos existiert. "What's Hecuba to him, or he to Hecuba, / That he should weep for her?" (SHAKESPEARE: 1984:152 / 2.2.553-554), fragt Hamlet sich an dieser prominenten Stelle. Er findet es verwunderlich, dass eine bloße Fiktion den Darsteller zum Erblassen und Weinen bringt. Denn es ist ja keine echte Leidenschaft, sondern nur ein Traum von Leidenschaft (,a dream of passion“1), der die Seele des Schauspielers derart beherrscht, dass sein Körper den Ausdruck des Leids annimmt. Allein durch die Vorstellung des Leids treten Tränen in seine Augen, Verzweiflung in seinen Gesichtsausdruck und die Stimme bricht. Die Körpersprache des Schauspielers wird allein von Imagination beherrscht. Was Hamlet uns hier vorführt, ist ein Erstaunen über die Professionalität des kalten Schauspielers, der auf der Bühne große Gefühle auf mitreißende Art präsentieren

1 ,Is it not monstrous that this player here,/ But in a fiction, in a dream of passion,/ Could force his soul so to his own conceit/ That from her working all his visage wann'd,/ Tears in his eyes, distraction in's aspect,/ A broken voice, and his whole function suiting/ With forms to his conceit? and all for nothing!" (SHAKESPEARE 1984:152 / 2.2.545-551) Hamlet überlegt weiter, wie es wäre, wenn der Schauspieler nun echte Leidenschaft fühlen würde, wenn er einen Grund für starke Emotionen hätte. Der Gefühlsschauspieler zöge laut Hamlet das Publikum noch massiver in Bann; er würde es rasend machen: „He would drown the stage with tears/ And cleave the general ear with horrid speech,/ Make mad the guilty and appal the free,/ Confound the ignorant, and amaze indeed/ The very faculties of eyes and ears“ (SHAKESPEARE 1984:152 / 2.2.556-561). 
kann, ohne sie selbst zu empfinden und ohne in der realen Welt einen Grund für sie zu haben.

Im Folgenden geht es um die Debatte um den heißen und den kalten Schauspieler, die im 18. Jahrhundert vor allem in Frankreich, England und Deutschland geführt wurde: Im ersten Schritt wird historisch skizziert, wie sich die konträren Schauspieltheorien in den Schriften von Sainte-Albine und Riccoboni herausbilden. Nach dieser historischen Erörterung werde ich in einem zweiten Schritt systematisch die Bedeutung der Kälte bei DIDEROT und LESSING herausarbeiten. Im dritten Schritt soll darauf aufbauend gezeigt werden, dass durch DiDEROTS und LESSINGS Ästhetik zwei verschiedene Arten von Aufklärung repräsentiert werden.

Die Theorien über die schauspielerische Darbietung im zeitgenössischen Theater etablieren sich um 1750 erstmals im ästhetischen Diskurs. Zwischen Frankreich, England und Deutschland findet eine explizite gegenseitige Bezugnahme statt. Die Figuren auf der Bühne repräsentieren zunehmend nicht mehr nur bestimmte Affekttypen, sondern entwickeln individuelle Persönlichkeiten mit emotionaler Komplexität. Die Natur des Menschen und das wahre Leben werden zum Vorbild, während die Künstlichkeit des klassizistischen Deklamationstheaters keine gültige Norm mehr darstellt. ${ }^{2}$

Der Hauptvertreter des heißen Schauspielers ist Pierre Rémond de SainteAlbine. Er ist Gelehrter und Journalist, kein Schauspieler, aber Theaterbesucher in Paris. Sainte-Albine verlangt vom Schauspieler, dass er tatsächlich empfindet, was er darstellt, also nicht nur spielt und vortäuscht. Seine zentrale Forderung lautet, dass diejenigen Empfindungen, die auf dem Theater gezeigt werden, von den Schauspielern selbst gefühlt werden, denn andernfalls sei das Bild der dargestellten Leidenschaften unvollkommen und niemals werde „die Kunst die Stelle der Empfindung ersetzen“. ${ }^{3}$ Sainte-Albine ist davon überzeugt, dass ein

2 Affekte galten als universell, nicht als individuell verschieden. Das ändert sich im Laufe des 18. Jahrhunderts: Gefühle als individuelle Zustände werden von Affekten unterschieden. Diese Individualisierung stellt neue Ansprüche an Schauspieler. Gefühle sind subtiler und verlangen eine Einfühlung in die Figur (vgl. RoselT 2017:97 und 44). Vgl. auch STEPHAN (1986:101): „Vor Mitte des 18. Jahrhunderts ist die Schauspielkunst noch nicht explizit Gegenstand kunsttheoretischer Betrachtungen."

„Wenn ihr diejenigen Empfindungen, die ihr uns auf dem Theater zeigen wollet, nicht selbst fühlet, so zeiget ihr sie uns nicht selbst, sondern nur ein unvollkommenes Bild davon, und niemals wird die Kunst die Stelle der Empfindung ersetzen“ (SAinTE-Albine 2017:102). 
kalter Schauspieler, der eine Rolle nur spielt, ohne mit ihr emotional identisch zu werden, die dargestellten Gefühle nicht überzeugend vermitteln kann. Er wirkt künstlich und berührt die Zuschauer kaum. ${ }^{4}$ Das heißt: Es ist die Aufgabe des Schauspielers, nicht nur zu spielen, sondern dem Gefühl nach zu sein, was er darstellt. Doch bei aller Identifikation mit seiner Rolle muss der Akteur letztlich Schau-Spieler bleiben, was zum Beispiel daran deutlich wird, dass die Schauspieler eine Verwandlungsfähigkeit besitzen sollen (vgl. SAINTE-ALBINE 2017:102). In verschiedene Rollen schlüpfen kann nur, wer sein Naturtalent mit Spieltechnik ausgebildet hat. Außerdem müssen Regeln der Schicklichkeit eingehalten werden. Sainte-Albine verlangt sogar eine Abmessung der „Gesticulation" (SAINTE-ALBINE 2017:111) ${ }^{5}$ nach nationalen Konventionen, d.h. auf deutschen Bühnen darf nicht so wild gestikuliert werden wie in Italien.

Zwei Aspekte möchte ich bei Sainte-Albine besonders hervorheben: Erstens, die Kausalität zwischen der Körpersprache des Schauspielers und seiner Empfindung sieht bei Sainte-Albine so aus, dass nur die wahre Empfindung einen überzeugenden und natürlichen Ausdruck hervorbringen kann. Dies ist festzuhalten, weil LESSING die Kausalität umkehren wird: Durch naturgetreue Nachahmung versetzt sich der Schauspieler bei LESSING in eine seelische Lage, die der Rolle, die er spielt, entspricht. Also geht bei Sainte-Albine die Kausalität von Innen nach Außen, bei LESSING von Außen nach Innen. Zweitens, die Wirkung auf das Publikum geht bei Sainte-Albine zwingend mit dem Gefühl des Schauspielers einher, das heißt, wenn er leidenschaftlich fühlt, reißt er seine Zuschauer entsprechend mit. Produktion und Rezeption verhalten sich wie eine „epidemische Krankheit“" (SAINTE-ALBINE 2017:106; vgl. STEPHAN 1986:106). Die Notwendigkeit dieser Kausalität wird bei LESSING ebenfalls angezweifelt.

Francesco Riccoboni fordert in seiner 1750 erschienenen Schrift L'Art du théâtre wie auch Sainte-Albine Natürlichkeit in der Darstellung. Aber in der Frage, wie dies zu erreichen ist, gehen die Theoretiker auseinander. Nach Riccoboni, der selbst Schauspieler in Frankreich war, hat jemand, der auf der Bühne tatsächlich empfindet, was er spielt, keine Kontrolle mehr über sein Spiel.

Der Zweck dieser Schauspieldoktrin ist es aber, das Publikum zu beherrschen. Als Beleg für den Zweck der Wirkung auf den Zuschauer siehe folgende Stellen bei Sainte-Albine: „Die Hauptregel ist, setze deine Zuschauer in Bewegung“ (SAINTEAlBINE 2017:104); „Dies sind die Gebiether, die als unumschränkte Herren über unsere Seelen herrschen“ (SAINTE-ALBINE 2017:105).

Auch bei Riccoboni gelten trotz der geforderten Natürlichkeit Regeln, die zeitgenössischen Konventionen entsprechen (z.B. dass die Hand nicht höher gebracht werden darf als auf Augenhöhe (Vgl. RicCOBONi 2017:114). 
Explizit weist er die Forderung nach dem heißen Schauspieler zurück, indem er zwei Argumente anführt: Erstens beherrscht jemand, der von Gefühlen überwältigt ist, sein Spiel nicht: Ihm erstickt die Stimme, er gerät in ein „lächerliches Stocken“ (RICCOBONI 2017:117). Und dazu gehört, zweitens, dass er auch nicht imstande ist, einen jähen Wechsel von Zärtlichkeit zu Zorn zu vollziehen. Die Handlung macht eine hohe Geschwindigkeit von Gefühlswechseln erforderlich.

Und wenn der heiße Schauspieler zum raschen Empfindungswechsel gezwungen wäre? Nach Riccoboni hätte er keine Kraft dazu und würde „eine Zeitlang aufs höchste nur mechanisch spielen“ (RICCOBONI 2017:117). So scheint das mechanische Spiel genau das zu sein, was Riccoboni vom kalten, nämlich empfindungslosen Schauspieler verlangt. Aber der Schauspieler soll nicht kalt in dem Sinne sein, dass er selbst gar nichts empfindet. Er soll nur nicht das empfinden, was er spielt. Er soll die Leidenschaft seiner Figur schon „lebhaft bewegt" (RICCOBONI 2017:118) darstellen, allerdings soll die Lebhaftigkeit nicht aus der Eifersucht oder Verzweiflung kommen, die dargestellt wird, sondern aus der Anstrengung, eine solche Leidenschaft darzustellen, die der Schauspieler nicht empfindet.

So wie Sainte-Albine glaubt, die Zuschauer würden von der Leidenschaft, die der Schauspieler tatsächlich empfindet, unvermeidlich angesteckt, geht Riccoboni davon aus, dass das Publikum von der lebhaften Aktivität des reinen Spiels notwendig fortgerissen wird. In beiden Fällen werde das Publikum also begeistert, obwohl der Darsteller bei Sainte-Albine heiß und bei Riccoboni kalt sein muss.

Diese Heiß-Kalt-Metaphorik ist dankbar, weil sie uns den Gegensatz der beiden Positionen so klar vor Augen führt. Gemeinsamkeiten bestehen aber dennoch bei beiden Theorien: Erstens soll die Wirkung auf das Publikum bei beiden mitreißend sein. Es ist nicht so, dass die Wirkung des kalten Schauspielers auf das Publikum auch kalt sein soll, also etwa im Sinne einer Reflexion, die Brecht ${ }^{6}$ später vom Theaterpublikum fordern wird. Zweitens haben beide Theorien in ihrer Abgrenzung vom Deklamationstheater ein Ideal von Natürlichkeit.

6 Jauß zufolge habe DiDERots Paradoxe sur le Comédien „sein modernes Gegenstück in Bert Brechts Neuer Technik der Schauspielkunst, deren zahlreiche Entsprechungen eine vergleichende Untersuchung geradezu herausfordern“. Brechts Verfremdungseffekt setzt nach Jauß genau wie DiDEROTs Paradoxe die NichtIdentifikation des Schauspielers mit seiner Rolle voraus (vgl. JAUB 1961:412). Aber der Illusionsbruch bei Brecht, der Reflexion im Zuschauer hervorruft, ist ein wesentlicher Unterschied: Bei DIDEROT soll der Zuschauer emotional beherrscht werden und nicht reflektieren. 
Dazu gehört, dass auch die einfachen Leute nachgeahmt werden sollen, nicht nur edle Charaktere und Leute von hohem Stand. Drittens gilt dieses Natürlichkeitsideal in beiden Theorien nur mit Einschränkung: Sainte-Albine gibt Regeln der Darstellung vor wie die, dass Gestik und Mimik abwechslungsreich und dem Nationalcharakter angemessen sein sollen. Ähnliches macht Riccoboni geltend: Da gibt es z.B. die Regel, dass die Natürlichkeit nicht exakt nachgeahmt werden soll, wenn sie frostig oder wild wirken könnte. Das Spiel dürfe weder übertrieben noch unterkühlt sein. ${ }^{7}$

Nachdem die Gegensätze und die Gemeinsamkeiten der beiden Positionen deutlich geworden sind, wird es nun darum gehen zu zeigen, wie DIDEROT und LESSING sich in dieser Debatte positionieren. ${ }^{8}$ Der Schauspieler soll nach DIDEROT $^{9}$ die Gefühle, die er darstellt, nicht empfinden, sondern kontrolliert fingieren. ${ }^{10}$ Aus seinem assoziativen Dialog lassen sich sechs Argumente herausfiltern, mit denen er den kalten Schauspieler verteidigt.

Erstes Argument: Auf der Bühne gelten andere Regeln als in der Natur: „Und wie sollte die Natur auch ohne Kunst einen großen Schauspieler bilden, da sich

7 Wer Raserei mimt, könne den Ausdruck des Pöbels nachahmen, müsse aber ein anständiges Betragen beibehalten: „,Kurz, man muß sich ausdrücken wie der Pöbel und betragen wie Leute vom Stande“" (RICCOBONI 2017:119).

$\mathrm{Zu}$ Lessings Rezeption der Theatertheorien Riccobonis und Diderots vgl. GOLAWSKI-BRAUNGART (2005).

DIDEROTS Schrift Paradoxe sur le comédien ist 1830 postum veröffentlicht worden, entstanden zwischen 1773 und 1777 und hat eine Überarbeitung in Dialogform erfahren. Der Ursprungstext ist eine Rezension von 1769 mit dem Titel Obervation sur une brochure intitulée Garrick ou les acteurs anglais über Antonio Fabio Sticottis Buch Garrick ou les acteurs anglais. Dieses Buch ist eine Übersetzung von John Hill The Actor: a Treatise on the Art of Playing (1750) ins Französische. Hills Buch ist schon eine Übersetzung des französischsprachigen Buchs von SainteAlbine gewesen, angereichert mit Bezugnahmen auf englische Schauspieler. Sticotti hat also ohne Kenntnis von Sainte-Albine die Übersetzung dieses Textes rückübersetzt (vgl. ROSELT 2017:136).

Johannes Lehmann argumentiert gegen eine Positionierung DIDEROTS zugunsten des kalten Schauspielers: „All den Argumenten für den kalten Schauspieler, die auf der beschriebenen Kluft zwischen Bühne und Gesellschaft beruhen, wird durch die Kritik an ihr letztlich wieder der Boden entzogen“" (LeHMANN 2000: 238). Der auftretende Erzähler habe die Funktion, die Identifikation des Ersten mit DiDEROT zu verunsichern und den Leser auf sich selbst zu stoßen. Inwiefern der Erzähler durch sein bloßes Auftreten die Position des Ersten in Frage stellt, bleibt jedoch offen. 
auf der Bühne doch nichts ebenso abspielt wie in der Natur und da alle dramatischen Werke nach einem bestimmten Regelsystem geschaffen sind?"“(DIDEROT 1981:291) Und: „Übertragen Sie Ihren privaten Ton, Ihren einfachen Ausdruck, Ihre alltägliche Haltung, Ihre natürliche Geste auf die Bühne, und Sie werden sehen, wie arm und schwach sie sind“ (DIDEROT 1981:301).

Diese Regeln müssen eingehalten werden und dazu gehört, dass die Darbietung mit einem Idealbild übereinstimmt und keine naturalistische Nachahmung ist:

Heißt [Wahr-sein auf dem Theater] die Dinge so zeigen, wie sie in der Natur sind? Keineswegs! Das Wahre in diesem Sinne wäre nur das Alltägliche. Was also ist das Wahre auf der Bühne? Es ist die Übereinstimmung der Handlungen, Reden, der Gestalt, der Stimme, der Haltung, der Geste mit einem vom Dichter erfundenen Idealbild, das oft vom Schauspieler noch überbetont wird. (DIDEROT 1981:303)

Zweites Argument: Ein Schauspieler muss alle Rollen spielen können und nicht nur die, mit der er sich identifizieren kann. Dazu ist Verstand nötig, nicht Gefühl: „Darum fordere ich von ihm durchdringenden Verstand und keinerlei Gefühl, die Kunst, alles nachzuahmen, oder [...] eine gleiche Befähigung für alle möglichen Rollen und Charaktere“" (DIDEROT 1981:293).

Drittes Argument: Ein heißer Schauspieler kann seine Leistung nicht exakt wiederholen. Wenn die Darstellung von seiner Stimmung abhängt, spielt er auf unkontrollierbare Weise eine Aufführung glänzend und eine andere schwach:

Wenn der Schauspieler Gefühl hätte, könnte er - Hand auf Herz! - zweimal hintereinander die gleiche Rolle mit der gleichen Wärme und dem gleichen Erfolg spielen? [...] Erwartet wird von ihnen keinerlei einheitliche Wirkung, ihr Spiel ist abwechselnd stark und schwach, heiß und kalt, platt und erhaben. [...] Dagegen bleibt sich der Schauspieler, der mit Überlegung nach dem Studium der Natur in dauernder Nachahmung eines idealen Vorbildes aus der Phantasie, dem Gedächtnis spielt, immer gleich und vollkommen: alles war abgemessen, abgewogen, überlegt, geordnet worden in seinem Kopfe. (DIDEROT 1981:293 und 294)

Leistung und Wirkung sind beim heißen Schauspieler unbeständig. Der kalte Schauspieler hingegen beherrscht sein Spiel so, dass es präzise seiner Idee folgt und daher immer gleich, d.h. reproduzierbar, ist.

Viertes Argument: Die bewegende Wirkung auf das Publikum geht von dem Darsteller aus, der sich unter Kontrolle hat, nicht von dem, der leidenschaftlich und ekstatisch ist: „Nicht der erregte Mensch, der außer sich ist, kann uns mitreißen; das ist das Vorrecht des Menschen, der sich in der Gewalt hat" (DIDEROT 1981:296).

Fünftes Argument: Ein Schauspieler, der sehr bestürzt ist, kann nicht spielen: „Man sagt, daß man weint, aber man weint nicht, wenn man sich darum bemüht, 
einem Vers Harmonie zu geben: oder wenn die Tränen fließen, entfällt die Feder den Händen. Man gibt sich seinem Gefühl hin und hört auf, zu gestalten“ (DIDEROT 1981:319).

Wenn der Schauspieler zutiefst erschüttert wäre, argumentiert DIDEROT, „wie könnte man in die Garderobe gehen, das unmäßige Lachen eines Dritten unterbrechen und ihm sagen, es sei Zeit, auf die Bühne zu kommen, um sich zu erstechen?"“(DIDEROT 1981:309)

Sechstes Argument: Die Bühne fordert einen Stimmungswechsel binnen Sekunden. Der Gesichtsausdruck kann plötzlich Erstaunen auf Verzweiflung folgen lassen, aber die Seele, die wahrhaft empfindet, kommt so schnell nicht mit:

„Garrick steckt seinen Kopf durch einen Türspalt und im Laufe von vier bis fünf Sekunden verändert sich sein Gesichtsausdruck von wilder Freude über gemäßigte Freude zur Ruhe, von der Ruhe zur Überraschung, von der Überraschung zum Erstaunen, vom Erstaunen zur Trauer, von der Trauer zur Niedergeschlagenheit, von der Niedergeschlagenheit zum Schrecken, vom Schrecken zum Entsetzen, vom Entsetzen zur Verzweiflung. Von dieser letzten Stufe steigt er wieder zum Ausgangspunkt. Kann eine Seele all diese Gefühle empfinden und diese ganze Skala in Übereinstimmung mit dem Gesicht?" (DIDEROT 1981:314)

Alle sechs Argumente laufen darauf hinaus, dass der Schauspieler, der echt empfindet, was er darstellt, zu befangen ist, um den Anforderungen der Bühne gerecht zu werden. Zu diesen Anforderungen gehören allgemeine Bühnenregeln, die Reproduzierbarkeit der Darstellung sowie der schnelle Stimmungsund Rollenwechsel.

Die Argumente vier und fünf seien im Folgenden genauer erörtert, weil sie den Kern der Position DIDEROTS darstellen. Zuerst zum vierten Argument: Die ergreifende Wirkung auf das Publikum geht von dem kontrollierten, kalten Darsteller aus, nicht von dem heißen, der fühlt, was er mimt. Der Schauspieler soll kalt die menschliche Natur beobachten und auch die heißblütigsten Charaktere mit präzise erlernter Technik auf die Bühne bringen. Das Gefühl ist nicht seine Eigenschaft, sondern die intendierte Wirkung beim Publikum. Hier wird noch einmal deutlich, dass es das gemeinsame Ziel beider Positionen ist, das Publikum emotional anzusprechen und für sich zu gewinnen. Dies ist ein wirkungsästhetisches Ziel, das produktionsästhetisch in kalt und heiß divergiert. Der geniale Schauspieler ist nicht der Gefühlsmensch. DIDEROT bringt im Kontext dieses vierten Arguments den Geniegedanken ins Spiel, um ihn für den kalten Verstandesschauspieler zu beanspruchen. Das Genie ist demnach kein Naturtalent, das künstlerisch aus sich selbst heraus schafft, was es präsentiert. Das Genie wendet in DIDEROTS Sprachgebrauch auf professionelle Weise Regeln 
an, die der Kopf erlernt hat: „Nicht sein Herz, sondern sein Kopf tut alles“ (DIDEROT 1981:297). Der Gefühlsschauspieler hat immer Schwächen, während der Verstandesschauspieler absolut kontrolliert - und insofern genial - nur den Ausdruck des Gefühls nachahmt und diesem somit nicht unterliegen kann: ,[S]ein ganzes Talent besteht nicht, wie ihr annehmt, im Fühlen, sondern in der Fähigkeit, die äußeren Zeichen des Gefühls so gewissenhaft wiederzugeben, daß ihr euch täuschen laßt" (DIDEROT 1981:299). Genie ist also das Produkt von Studium, Technik, Präzision, Gedächtnisleistung und Verstandesregeln - also kalten Eigenschaften. Damit höhlt DIDEROT auf aufklärerisch rationalistische Weise die Naturtalent-Anteile des Geniegedankens aus.

Nun zum fünften Argument: Ein Schauspieler, der sehr bestürzt ist, kann nicht spielen. Ähnliches hat Riccoboni geltend gemacht. Der empfindende Schauspieler ist emotional unbeherrscht, er komponiert sein Spiel nicht. Ihm zufolge müssten echte Gefühle sogar dazu führen, reale Gewalt auf der Bühne anzuwenden: „Da sieht man ja einen Menschen, welcher von seiner Rolle so durchdrungen zu sein scheint, dass er sogar die Raserei empfindet! Aber warum tötete er denn niemals einen Schauspieler, welcher mit ihm spielte?" (RICCOBONI 2017:118) Riccoboni schließt das Empfinden mit dem Handeln des Schauspielers kurz, um zu zeigen, dass wahre Empfindungen auf der Bühne fehl am Platze sind. DIDEROTS Diktum, wer bestürzt sei, könne nicht spielen, liegt auf dieser Linie, denn das Spielenkönnen ist gebunden an ein Bewusstsein der Fiktionalität.

LESSING sind die Positionen Riccobonis, Sainte-Albines und DIDEROTs durch seine Übersetzungen sehr gut bekannt. Während DIDEROT sich auf die Seite des kalten Schauspielers schlägt, nimmt LESSING eine Position ein, die etwas differenzierter und komplexer ist. Im Zweifelsfall hat zwar auch der kalte Schauspieler bei LESSING Vorrang, aber er sieht in der kalten Darstellung keine Garantie dafür, dass Mitleid mit dem Helden auf der Bühne erzeugt wird. Wodurch sich LESSING von den anderen Positionen unterscheidet, ist, dass er den unmittelbaren Zusammenhang zwischen der Empfindung des Schauspielers und der Wirkung auf das Publikum in Frage stellt. Als Beleg dafür, dass LESSING echte Gefühle auf der Bühne bejaht, sei die folgende Stelle aus der Hamburgischen Dramaturgie angeführt: „Wie weit ist der Akteur, der eine Stelle nur versteht, noch von dem entfernt, der sie auch zugleich empfindet!" (LESSING 2010:197) ${ }^{11}$ LESSING argumentiert, dass die Seele des Schauspielers ganz gegenwärtig bei der Rolle sein muss, weil er sonst mechanisch redet wie ein Papagei. Der

11 Die folgenden Lessing-Zitate stammen aus dem 3., 4. und 5. Stück der Hamburgischen Dramaturgie. 
Schauspieler soll die Sätze nicht mühsam aus seinem Gedächtnis auskramen; seine Worte und Gesten sollen vielmehr ,unmittelbare Eingebungen der gegenwärtigen Lage“ sein; er soll „den ganzen Sinn seiner Worte durchdrungen“ haben (LESSING 2010:197). Was spricht nun gegen den heißen Schauspieler, dessen Hitze LESSING ja offenbar begrüßt? Es ist nicht gewährleistet, dass das, was der Schauspieler empfindet, auch entsprechend auf das Publikum wirkt: „Aber auch dann kann der Akteur wirklich viel Empfindung haben, und doch keine zu haben scheinen“ (LESSING 2010:197). Innen und Außen können LESSINGS Bedenken zufolge dergestalt auseinanderklaffen, dass das Publikum den Akteur nicht überzeugend findet, obwohl er echt fühlt, was er spielt:

Denn die Empfindung ist etwas Inneres, von dem wir nur nach seinen äußern Merkmalen urteilen können. Nun ist es möglich, daß gewisse Dinge in dem Baue des Körpers diese Merkmale entweder gar nicht verstatten, oder doch schwächen und zweideutig machen. Der Akteur kann eine gewisse Bildung des Gesichts, gewisse Mienen, einen gewissen Ton haben, mit denen wir ganz andere Fähigkeiten, ganz andere Leidenschaften, ganz andere Gesinnungen zu verbinden gewohnt sind, als er gegenwärtig äußern und ausdrücken soll. Ist dieses, so mag er noch so viel empfinden, wir glauben ihm nicht: denn er ist mit sich selbst im Widerspruche. (LESSING 2010:197)

Daher hält LESSING den kalten Schauspieler für theatertauglicher, weil dieser in der Körpersprache treffsicher ist, auch wenn er nichts empfindet. Ihm gelingt es, beseelt zu erscheinen, auch wenn er nur mechanisch nachäfft:

Gegenteils kann ein anderer so glücklich gebauet sein; er kann so entscheidende Züge besitzen; alle seine Muskeln können ihm so leicht, so geschwind zu Gebote stehen; er kann so feine, so vielfältige Abänderungen der Stimme in seiner Gewalt haben; kurz, er kann mit allen zur Pantomime erforderlichen Gaben in einem so hohen Grade beglückt sein, daß er uns in denjenigen Rollen, die er nicht ursprünglich, sondern nach irgendeinem guten Vorbilde spielet, von der innigsten Empfindung beseelet scheinen wird, da doch alles, was er sagt und tut, nichts als mechanische Nachäffung ist. Ohne Zweifel ist dieser, ungeachtet seiner Gleichgültigkeit und Kälte, dennoch auf dem Theater weit brauchbarer, als jener. (LESSING 2010:198)

Doch mit dieser Alternative gibt LESSING sich nicht zufrieden. Er fordert explizit eine „Mischung“ aus „Feuer und Kälte“: ,Folglich mit Begeisterung und Gelassenheit, mit Feuer und Kälte? - Nicht anders; mit einer Mischung von beiden, in der aber, nach Beschaffenheit der Situation, bald dieses, bald jenes hervorsticht"“ (LESSING 2010:199).

Der Akteur wird durch sein Spiel dahin gebracht, sich in den Zorn, den er darstellt, hineinzuversetzen, so dass der Körper den rechten Ausdruck des Zorns annehmen wird: 
Und ich sage; wenn er nur die allergröbsten Äußerungen des Zornes einem Akteur von ursprünglicher Empfindung abgelernet hat und getreu nachzumachen weiß - den hastigen Gang, den stampfenden Fuß, den rauhen, bald kreischenden bald verbissenen Ton, das Spiel der Augenbraunen, die zitternde Lippe, das Knirschen der Zähne usw. - wenn er, sage ich, nur diese Dinge, die sich nachmachen lassen, sobald man will, gut nachmacht: so wird dadurch unfehlbar seine Seele ein dunkles Gefühl von Zorn befallen, welches wiederum in den Körper zurückwirkt. (LESSING 2010:199)

Während bei Sainte-Albine der körpersprachliche Ausdruck zwangsläufig aus der echten Empfindung hervorgeht, dreht LESSING die Kausalität um: Der Schauspieler ahmt sozusagen kalt nach und wird dabei heiß. Damit ist die Kontrolle über das Spiel gewährleistet, wie DIDEROT sie fordert. Zugleich ist der Schauspieler ein beseelter Mensch, der in seinem Spiel emotional ganz gegenwärtig ist. Es kann nach LESSING gar nicht das Problem sein, dass der Schauspieler zu viel Feuer hat:

$[\mathrm{M}]$ an erstreitet sich so sehr, ob ein Schauspieler zu viel Feuer haben könne. Wenn die, welche es behaupten, zum Beweise anführen, daß ein Schauspieler ja wohl am unrechten Orte heftig, oder wenigstens heftiger sein könne, als es die Umstände erfodern: so haben die, welche es leugnen, recht zu sagen, daß in solchem Falle der Schauspieler nicht zu viel Feuer, sondern zu wenig Verstand zeige. (LeSSING 2010:209)

Was macht LESSING hier? Er nimmt der Kritik am Empfindungsschauspieler den Wind aus den Segeln, indem er sagt, dass die Empfindung dem mitreißenden Schauspiel gar nicht im Weg stehe. Denn Feuer bedeute nicht wildes „Geschrei“, sondern eine „Lebhaftigkeit“, die bühnentauglich gesteuert werden müsse:

Überhaupt kömmt es aber wohl darauf an, was wir unter dem Worte Feuer verstehen. Wenn Geschrei und Kontorsionen Feuer sind, so ist es wohl unstreitig, daß der Akteur darin zu weit gehen kann. Besteht aber das Feuer in der Geschwindigkeit und Lebhaftigkeit, mit welcher alle Stücke, die den Akteur ausmachen, das ihrige dazu beitragen, um seinem Spiele den Schein der Wahrheit zu geben: so müßten wir diesen Schein der Wahrheit nicht bis zur äußersten Illusion getrieben zu sehen wünschen, wenn es möglich wäre, daß der Schauspieler allzuviel Feuer in diesem Verstande anwenden könnte. (LESSING 2010:210)

Diese Steuerung der Gefühle ist wichtig im Hinblick auf die Publikumswirkung, denn unkontrollierte Gefühlsausbrüche erwecken keine Sympathie beim Zuschauer, sondern Missbilligung.

In diesem Punkt ist eine Parallele Lessings zu Adam Smith' Theory of Moral Sentiments zu sehen, durch die deutlich wird, was die Kälte bei LESSING leistet und von der geforderten Kälte bei DIDEROT unterscheidet. Obwohl es zwischen 
LESSING und DIDEROT Gemeinsamkeiten gibt - beide verlangen ein kontrolliertes Spiel ohne Gefühlsausbrüche und sehen darin die zu erzielende packende Wirkung auf das Publikum gesichert - kann DIDEROT keinen Gewinn darin sehen, wenn der Schauspieler durch seine Darstellung echte Empfindungen seiner Rolle entwickelt. LESSING auf der anderen Seite begrüßt das Feuer des Akteurs, fordert aber eine gewisse Kälte, welche die Flamme reguliert. Bei LESSING sorgt die Kälte dafür, dass das Publikum nicht mit Heftigkeiten überfordert wird, denn es soll ja Mitgefühl entwickeln und sich nicht vom Helden befremdet fühlen. LESSINGS Appell für Kälte liegt demnach auf der aufklärerischen Linie von Adam Smith, derzufolge moralische Gefühle im Mitleiden entstehen können, sofern das gezeigte Leiden maßvoll und nachfühlbar ist. LESSING hat im Laokoon (1766) Smith' Theory of Moral Sentiments (1759) explizit kommentiert. Der schottische Aufklärungsphilosoph macht geltend, dass unser moralisches Urteil über andere davon abhängt, inwieweit wir uns in seine Empfindungen hineinversetzen können. Mitleid empfinden wir dann, wenn die Gebärden des Anderen Leiden ausdrücken, ohne dass diese allzu körperlich und unverhältnismäßig sind. Wir billigen sein Verhalten, sofern es nicht unangemessen ist, und sofern wir uns in seine Lage versetzen können:

When we judge in this manner of any affection, as proportioned or disproportioned to the cause which excites it, it is scarce possible that we should make use of any other rule or canon but the correspondent affection in ourselves. If, upon bringing the case home to our own breast, we find that the sentiments which it gives occasion to, coincide and tally with our own, we necessarily approve of them as proportioned and suitable to their objects; if otherwise, we necessarily disapprove of them, as extravagant and out of proportion. (SMITH 2000:17f.)

Smith entwickelt keine Theatertheorie, aber die Empathie des Zuschauers mit dem Helden auf der Bühne kann analog beurteilt werden. Und so wird deutlich, was bei LESSING die Kälte des Schauspielers leistet: durch sie werden die Leidenschaften des Helden emotional nachvollziehbar; und damit wird die Empathiefähigkeit des Zuschauers ausgebildet.

Die Kälte steht bei LESSING also im Dienst der Moral und auf der Traditionslinie der aufklärerischen Moralphilosophie. Kälte ermöglicht eine Regulierung der Emotionen auf ein nachvollziehbares Maß, damit ein Mitgefühl ermöglicht wird, das den Zuschauer zu einem besseren, gerechteren, wohltätigeren Menschen macht. LESSING plädiert nicht für den kalten Schauspieler, sondern für eine Mischung aus Herz und Kopf, Feuer und Kälte. Er sagt einerseits: „Jede Moral [...] will mit einer gewissen Kälte gesagt sein“ (LESSING 2010:199). Und andererseits: „Alle Moral muss aus der Fülle des Herzens kommen“ (LESSING 2010:197). Das ist sozusagen LESSINGS Paradox über den Schauspieler: dass 
ihm beides abverlangt werden muss. Und es wird deutlich, wie und warum das so sein soll: Das Feuer garantiert „Licht und Leben“(LESSING 2010:204), also Beseeltheit gegenüber dem sentenzenhaften Aufsagen, und die Kälte ist dazu da, den „Affekt in Raisonnement sich auskühlen“ zu lassen (LESSING 2010:200).

Bei DIDEROT hingegen steht die Kälte nicht im Dienst der Moral. Der ,sangfroid diderotien' dient allein der praktischen Kunstfertigkeit der Darstellung, welche eine Reproduzierbarkeit sowie eine Kontrolle über das Schauspiel und dessen Wirkung auf das Publikum gewährleistet. Die Wahrheit der Nachahmung erfolgt nicht über emotionale Identifikation, sondern über ein kaltes Studium der menschlichen Natur. DIDEROTS Ziele sind auch aufklärerisch, aber weder moralisch noch empathiefreundlich. Aufklärerisch sind zwei Karten, die er ausspielt, erstens die Verstandesherrschaft, zweitens die handwerkliche Gemachtheit des Schauspiels. Es soll eine mitreißende Wirkung beim Publikum erzielt werden, und das Mittel zu diesem Zweck ist der kalte, technisch professionelle Schauspieler, und nicht der heiße, der seinen spontanen Eingebungen folgt. Nur das kalkulierte, handwerklich gemachte Spiel ist nach DIDEROT genial. ${ }^{12}$ Die moralische Wirkung auf das Publikum spielt dabei keine Rolle.

In einem kürzlich erschienenen Sammelband wird DIDEROTS Schauspielertext politisch und moralisch gedeutet. Dies ist $\mathrm{m}$. E. nicht überzeugend. Beispielsweise wird es als aufklärerisch bezeichnet, dass DIDEROT vor der manipulativen Kraft der Gefühle warnt (vgl. ABBT / FESTL 2018:63). In Wahrheit äußert sich DIDEROT in dem Text aber nicht skeptisch gegenüber einer Manipulation des Publikums mittels Emotionen. Im Gegenteil, die Gefühlsherrschaft über das Publikum soll der Schauspieler ja unbedingt erzielen. Er soll Meister der Geister werden. DIDEROT verwirft dabei die Empfindungen, weil er sie für dilettantisch und unverlässlich hält - weil sie also gerade nicht zuverlässig ,manipulieren'. In diesem Punkt geht es DIDEROT gar nicht um Moral - und auch nicht, mit Schiller gesprochen, um die republikanische Freiheit des Rezipienten, die ja ebenfalls moralisch begründet ist.

Als Ausblick sei zum Schluss eine Linie in die Gegenwart skizziert: Die Verfechter des kalten Schauspielers sagen, dass echte Gefühle auf der Bühne nichts zu suchen haben. Riccoboni behauptet sogar, dass ein Schauspieler, wenn er

12 Damit steht DiDEROT quer zum irrationalistischen Geniebegriff seiner Zeit, insofern die Geniezeit sich im Namen schöpferischer Spontaneität gegen die ,Regeln' auflehnt. Vgl. zum Geniekonzept ScHMIDT (1985:82). 
zum Beispiel echte Raserei empfinden würde, eigentlich seinen Mit-Schauspieler auf der Bühne töten müsste. Und wenn er das nicht täte, dann sei seine Raserei auch nicht echt:

Da sieht man ja einen Menschen, welcher von seiner Rolle so durchdrungen zu sein scheint, dass er sogar die Raserei empfindet! Aber warum tötete er denn niemals einen Schauspieler, welcher mit ihm spielte? [...] Seine Raserei war also nicht wahr, weil sie seiner Vernunft die Freiheit zu wählen ließ. (RICCOBONI 2017:118)

Diese Folgerung, dass Gefühle nur dann echt sind, wenn sie durch reale Umstände ausgelöst werden, ist aus der Sicht der heutigen Emotionsforschung nicht haltbar. Zwar vertreten auch einige Philosophen des 20. Jahrhunderts noch die Position, dass Emotionen, die wir angesichts von fiktionalen Erzählungen empfinden, keine echten Emotionen seien. Kendall Walton z.B. argumentiert, dass man sich mit Fiktionalität auf ein ,game of make-belief" einlasse und nur Quasi-Gefühle, also z.B. ,quasi-fear“" angesichts einer Gefahr innerhalb der Geschichte empfinden könne (WALTON 1978:10 und 6). Es ist jedoch inzwischen neurologisch erwiesen, dass unser Gehirn reale von virtuellen Auslösereizen kaum unterscheiden kann (vgl. VOLAND 2007:8). Es wird dasselbe Emotionsprogramm initialisiert, ohne dass eine Handlung freigesetzt wird (vgl. MELLMANN 2006:149). In der gegenwärtigen Emotionsforschung besteht ein breiter Konsens darin: Das Wissen um Fiktionalität schwächt nicht die Emotionen ab, sondern reguliert nur die Verhaltensreaktion. ${ }^{13}$ Dasselbe gilt fürs Theater, und zwar sowohl für das Publikum als auch für die Akteure. Der heiße Schauspieler kann echt wütend sein, ohne seinen Mitspielern echten Schaden zuzufügen. Und der kalte Schauspieler kann mit seinem bloßen „dream of passion“ vollkommen fesselnd sein und beim Publikum echte Gefühle des Mitleids um Hekuba auslösen, die er selbst nicht empfindet. Dieses Ziel soll sowohl bei DIDEROT als auch bei LESSING erreicht werden. Ob es besser heiß oder kalt erreicht werden kann, bleibt bis heute eine unabgeschlossene Debatte.

13 „Grodal erklärt die emotionale visuelle Wirkung des Films neuropsychologisch mit der identischen Beschaffenheit und Intensität der Eindrücklichkeit realer und fiktionaler visueller Reize: In beiden Fällen werde im Gehirn die gleiche lokale Realität (local reality) abgebildet, während die Einschätzung der Reize als fiktional im Rahmen von Denkprozessen vorgenommen werde, die Grodal als global (global reality) bezeichnet“ (PALMIER 2014:78). 
Was leistet die Kälte des Schauspielers bei Lessing und Diderot?

\section{Literatur}

Abbt, Christine / Festl, Michael G. (2018) (eds.): Politik, Schauspiel, Philosophie. Eine Auseinandersetzung mit Denis Diderots Paradox über den Schauspieler. Studia Philosophica Vol. 77. Schweizerische Zeitschrift für Philosophie.

Diderot, DENis (1981): Erzählungen und Gespräche. Aus dem Französischen von Katharina Scheinfuß. Leipzig.

Golawski-Braungart, JutTA (2005): Die Schule der Franzosen. Zur Bedeutung von Lessings Übersetzungen aus dem Französischen für die Theorie und Praxis seines Theaters. Tübingen / Basel.

JAUß, HANS ROBERT (1961): Diderots Paradox über das Schauspiel (Entretiens sur ,Le Fils Naturel '). In: Germanisch-Romanische Monatsschrift, NF, XI, 380-413.

LeHMAnN, JoHANnES Friedrich (2000): Der Blick durch die Wand. Zur Geschichte des Theaterzuschauers und des Visuellen bei Diderot und Lessing. Freiburg.

Lessing, GotThOld EPHRAim (2010): Minna von Barnhelm/Hamburgische Dramaturgie. Text und Kommentar, ed. BoHnEN, KLAUS. Berlin.

Mellmann, Katja (2006): Literatur als emotionale Attrappe: eine evolutionspsychologische Lösung des „,paradox of fiction “. In: KLEIn, UtA / MELlmann, KATJA / METZGER, STEFFANIE (eds): Heuristiken der Literaturwissenschaft: disziplinexterne Perspektiven auf Literatur. Paderborn, 145-166.

PALmier, JeAn-PIERre (2014): Gefühlte Geschichten. Paderborn.

RiCCOBOni, FrAnCESCO (2017): Die Schauspielkunst. In: RoSELt, JENS (ed.): Seelen mit Methode - Schauspieltheorien vom Barock bis zum postdramatischen Theater. Mit einer Einführung von Jens Roselt. Berlin, 116-123.

Roselt, Jens (2017) (ed.): Seelen mit Methode - Schauspieltheorien vom Barock bis zum postdramatischen Theater. Mit einer Einführung von Jens Roselt. Berlin.

SAINTE-Albine, Pierre RÉmond de (2017): Der Schauspieler. In: Roselt, Jens (ed.): Seelen mit Methode - Schauspieltheorien vom Barock bis zum postdramatischen Theater. Mit einer Einführung von Jens Roselt. Berlin, 102-111.

SCHMIDT, Jochen (1985): Die Geschichte des Genie-Gedankens in der deutschen Literatur, Philosophie und Politik 1750-1945: Bd. 1: Von der Aufklärung bis zum Idealismus. Darmstadt.

ShaKeSPeARe, WiLliam (1984): Hamlet. Englisch/Deutsch, ed. und aud dem Englischen übers. von KLEIN, Holger M. Stuttgart.

Smith, ADAm (2000): The Theory of Moral Sentiments. New York.

STEPHAN, ULRIKE (1986): Gefühlsschauspieler und Verstandesschauspieler. Ein theatertheoretisches Problem des 18. Jahrhunderts. In: KÖRNER, HANS / PERES, CONSTANZE / Steiner, ReinHARd / TAVERnier, Ludwig (eds.): Empfindung und Reflexion. Ein Problem des 18. Jahrhunderts. Hildesheim / Zürich / New York, 99-116. 


\section{Susanne Kaul}

VOLAND, ECKART (2007): Virtuelle Welten in realen Gehirnen. Evolutionspsychologische Aspekte des Umgangs mit Medien. In: LiLi 146:7-22.

Walton, Kendall L. (1978): Fearing Fictions. In: The Journal of Philosophy 75:5-27. 